\title{
Kinetic masks: a new approach and device for dispersing biologically relevant fluids
}

\author{
Ina Meiser • Stephen Graham Shirley • \\ Heiko Zimmermann
}

Received: 2 April 2009/Accepted: 19 June 2009/Published online: 11 July 2009

(C) The Author(s) 2009. This article is published with open access at Springerlink.com

\begin{abstract}
The controlled dispersion of fluids, particularly biologically relevant solutions in micro-volumes, is of high practical interest in biotechnology and medicine. Pharmaceutical test assays, for example, need a method for the fast and defined deposition of fluid samples. Most current micro-dispensing methods, i.e. contact-based pin printing, have problems such as time delays, limited dosing velocity, minimum volume or high interference that limit biological applications. Spraying techniques suffer from a lack of reproducibility; a defined deposition of samples on targets is not possible. Here, we introduce a new method for the parallel and spatially defined dispersion of many microvolumes that overcomes disadvantages of common microdispensers. The overall approach is that a fluid drop, produced by a droplet generator, falls on a free trajectory with a defined kinetic energy, and is split by a masking unit placed perpendicular to the flight direction into at least two smaller droplets (Zimmermann et al. in Method and device for dosing fluid media, WO/2002/102515, Germany, 2002). On the target, the resulting droplets form reproducible patterns, which are enlarged and scalable images of the grid pattern. Possible applications for this method are noncontact cell patterning, cell encapsulation, cryopreservation and fast mixing processes in micro-volumes. Here, we use
\end{abstract}

I. Meiser · S. G. Shirley · H. Zimmermann ( $\square)$ Department for Biophysics and Cryotechnology, Fraunhofer Institute for Biomedical Engineering, Ensheimer Strasse 48, 66386 St. Ingbert, Germany

e-mail: heiko.zimmermann@ibmt.fraunhofer.de

H. Zimmermann

Department for Molecular and Cellular Biotechnology/

Nanotechnology, Saarland University, PO Box 1511 50, 66041

Saarbrücken, Germany this method for the direct and defined parallel positioning of cell suspensions on specific substrates, which can be useful for test assays, tissue engineering and cryopreservation.

\section{Introduction}

Modern life science and pharmaceutical and chemical research relies on high throughput techniques and high throughput screening methods. In particular, automation, miniaturisation and parallelisation decrease cost and sample volumes and allow faster analysis times (Lin et al. 2001; Gutmann et al. 2004; Chin et al. 2007). A key step is the dispersion of micro-volumes. Current micro-dispensing technologies (each with its own disadvantages for biologically relevant fluids) are bubble-jet printing (Okamoto et al. 2000; Allain et al. 2001; Cooley et al. 2002; Allain et al. 2004), piezoelectric actuators (Laurell et al. 1999; de Heij et al. 2003), stamping (Lin et al. 2001) and pneumatic actuators (Koltay et al. 2004). In pin printing technology, for example, pin reloading and the intermediate washing steps cause low dose rates and uncontrollable incubation times. To overcome the latter, multiple capillary systems were invented. However, they tend to be unreliable and have a minimum dispensed volume (Gutmann et al. 2004). These problems can be a hindrance with some reagents or assays.

In terms of handling biologically relevant fluids, the dispensing applications are usually limited to patterning small adhesive molecules, such as poly-L-lysine, or to protein micro-patterns that are generated to guide cell adhesion and growth (Roth et al. 2004; Gustavsson et al. 2007). No previous dispensing method is capable of defined parallel dispensing of cell suspensions. 
Dispensing cell suspensions requires the minimisation of shear and impact forces that might damage cells and well-defined deposition of drops with specific volumes (Thielecke et al. 2005).

To overcome the problems of uncontrollable incubation times and incapability of dispensing cells directly, we introduce the process of "kinetic masking". This new method allows the defined, fast, parallel and direct dispersing of biologically relevant fluids. This study describes the basic setup, applications and proof of concepts, as well as possibilities and limitations of this method.

\section{Materials and methods}

\subsection{Setup}

The setup is illustrated in Fig. 1a. A syringe pump (Sp 270, World Precision Instruments, Berlin, Germany) serves as a drop generator (see Fig. 1 flag 1). The pump's syringe contains the sample and is connected to a blunt cannula via either a Teflon tube with an inner diameter of $3.5 \mathrm{~mm}$ or an infusion tube (Heiland Med, Hamburg, Germany) with an inner diameter of $0.5 \mathrm{~mm}$. Cannulas had inner diameters: $0.4,1.2$ or $2.7 \mathrm{~mm}$ (KNLL syringe needles, ILS, Stützenbach, Germany).

The masking unit is connected to the drop generator by a downpipe of clear Plexiglas $(10,20,40,60$ or $110 \mathrm{~cm}$ length; $6 \mathrm{~cm}$ inner diameter), allowing every stage of the process to be observed by a high-speed video camera (SpeedCam Visario LT400, Weinberger Vision, Erlangen, Germany). The downpipe was treated with a commercial anti-static spray. The light sources are two ultra-high performance lamps (ILP-1, Olympus, Hamburg, Germany) and are sufficient for exposure rates up to 4,000 frames per second.

The masking unit (see Fig. 1b), which consists of a mount and masks, was designed and produced by Climaco Formenbau (St. Ingbert, Germany). The masks are either stainless steel (4301 VA perforated by laser, see Fig. 1c2, c3) or die-cast high-density polyethylene (HDPE produced by Climaco, see Fig. 1c1). The slot in the mount and the matching die-cast masks are tight fitting, so that the desired grid can be selected with one movement of the thumb and grid's position is automatically identical.
Fig. 1 a Schematic

representation of the setup and principle of the method. A droplet of defined size is generated by a syringe pump (1) and a blunt cannula (2). The height of fall (distance $d_{1}$, range $10-110 \mathrm{~cm}$ ) determines the kinetic energy of the drop. To prevent draughts and contamination, the falling drop is protected by a plexiglass downpipe. Distance $d_{2}$ between the masking unit (3), that holds the grid (4), and the substrate, affects scaling of the resulting droplet pattern (see Fig. 4). $d_{2}$ can be varied from 1 to $20 \mathrm{~cm}$. b Masking unit to hold grids (4) of different shapes and materials. c1 HDPE mask with different, easy exchangeable grids, produced by die casting with grid sizes from 200 to $1,200 \mu \mathrm{m}$. c2, c3 Metal (stainless steel $4301 \mathrm{VA}$, lasered) masks with grid size of $800 \mu \mathrm{m} \mathrm{(c2)} \mathrm{and} \mathrm{hole} \mathrm{diameter}$ of $1,000 \mu \mathrm{m}$, respectively

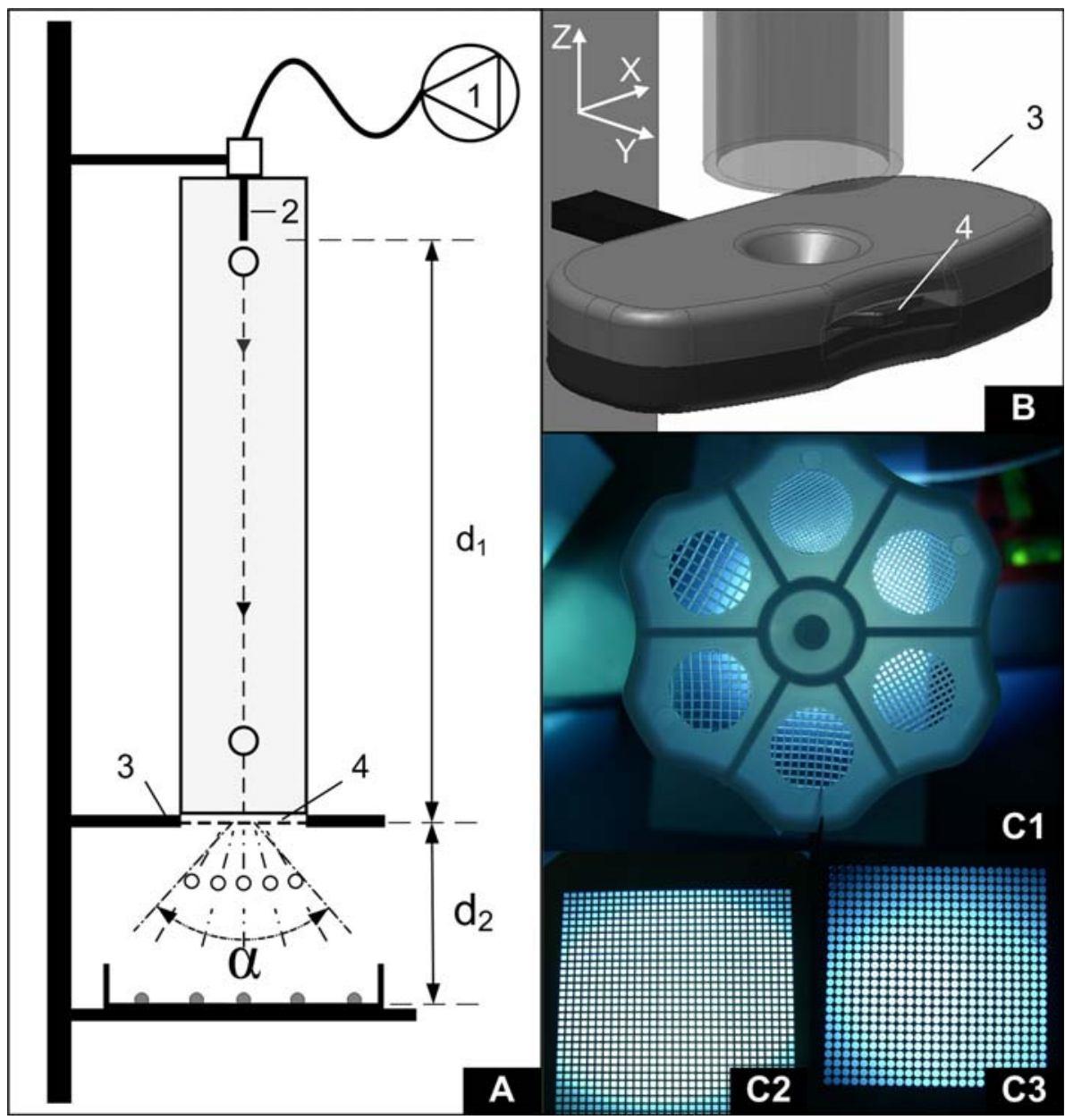


A feature of the HDPE masks is that one side of the grid's bar surface is plane, whereas the other side is slightly rounded. Typically, the grids are 350 (metal) to 500 (HPDE) $\mu \mathrm{m}$ thick. Figure $1 \mathrm{c} 1$ shows a HDPE mask with six grids of different sizes (holes are 200-1,200 $\mu \mathrm{m}$ in $200 \mu \mathrm{m}$ steps), another mask offers grids with different, non-regular structures (picture not shown). The mount is polyoxymethylene $\left(\right.$ Delrin $^{\circledR}$ ), a biocompatible thermoplastic adequate for high-precision components, due to its high stiffness, low friction coefficient and excellent dimension stability in a wide temperature range. For easy cleaning and sterilisation, the mount is made as two pieces, which can be screwed together.

The target mount holds a $10 \mathrm{~cm}$ diameter Petri dish and can be positioned automatically (accurate to $10 \mu \mathrm{m}$ ) by a computerised control panel (Cimrex 12, Beijer Electronics, Stuttgart, Germany). A metallic mirror (Laser 2000, Wessling, Germany) mounted below the dish at $45^{\circ}$ gives the camera an additional worm's eye view of the process. The high-speed videos are captured by Visart software (Weinberger Vision, Erlangen, Germany) and processed with IrfanView, ImageJ and Adobe Photoshop CS2. The target dish is in a humid environment to prevent dehydration and cell death.

Initial experiments were performed with ultra-pure water (Nanopure Diamond Water System; (Werner, Reinstwassertechnik, Leverkusen, Germany) dyed with trypan blue (Sigma, Schelldorf, Germany) or phenol red (AppliChem, Darmstadt, Germany) to improve contrast.

\subsection{Alginates and cross-linking solution}

Ultra-high viscosity alginates of clinical grade (Grohn et al. 1997; Hillgärtner et al. 1999) were prepared from the dried algae Lessonia nigrescens and Lessonia trabeculata, harvested at the Chilean coast and processed at Biozentrum Universität Würzburg. They were dissolved at 0.35 or $0.7 \%$ in isoosmolar $0.9 \% \mathrm{NaCl}$ (Merck, Darmstadt, Germany). To reduce the viscosity of the high-quality alginates by breaking long polysaccharide chains, the solutions were exposed to ultrasound for 3-15 min at Biozentrum Universität Würzburg.

Alginate was cross-linked (Zimmermann et al. 2007) with $20 \mathrm{mM} \mathrm{BaCl}{ }_{2}$ (Sigma, Schelldorf, Germany), $115 \mathrm{mM} \mathrm{NaCl}$ and $5 \mathrm{mM}$ histidine (Merck, Darmstadt, Germany) in ultra-pure water.

\subsection{Cell culture}

Murine fibroblast cells (L929 cell line) were obtained from DSMZ (Braunschweig, Germany) and cultivated with Dulbecco's modified Eagle media (Gibco, Invitrogen, Karlsruhe, Germany) comprising $10 \%$ foetal bovine serum and $1 \%$ Gentamycin in standard conditions $\left(37^{\circ} \mathrm{C}, 5 \%\right.$ $\mathrm{CO}_{2}$ ).

For cell suspension experiments, the adherent mouse fibroblasts were removed and concentrated to $2 \mathrm{E}+6$ cells/ $\mathrm{ml}$, then diluted 1:1 with alginate solution, cryoprotective solution [dimethyl sulfoxide (DMSO) or trehalose in culture medium] or culture medium.

For three-dimensional (3D) encapsulation, the kinetic mask product of $1 \mathrm{ml}$ alginate-cell mix was directed into a tank containing a cross-linking solution. After $20 \mathrm{~min}$ of incubation, the capsules were removed by centrifugation and surplus $\mathrm{BaCl}_{2}$ solution removed by three washes with PBS (Invitrogen, Karlsruhe, Germany). Vitality tests were performed immediately by fluorescence staining with ethidium bromide and fluorescein diacetate (Nikon fluorescence microscope Eclipse TE 300).

In 2D encapsulation, one droplet of a cell-alginate mixture was spotted by kinetic masking onto a target Petri dish pre-coated with $0.01 \%$ polylysine in PBS. The resulting droplet pattern was carefully overlaid with crosslinking solution, incubated for $20 \mathrm{~min}$ at RT and washed with PBS. The vitality of the encapsulated cells was determined by fluorescence staining. For comparison, 2D encapsulation studies were also performed with a Nanoplotter (NP2; GeSim, Dresden, Germany) (Ehrhart et al. 2007).

Some HDPE masks were treated with oxygen plasma (low pressure) in a "nano" system from Diener Electronic (Nagold, Germany) for 60-90 s to change the surface characteristics.

\section{Results}

\subsection{System handling}

In the case of ultra-pure water, dyed with trypan blue, cannulas with inner diameters of $0.41,1.2$ and $2.7 \mathrm{~mm}$ produce drops with 1.8, 2.4 and $2.8 \mathrm{~mm}$ diameter. For water (viscosity $\sim 1 \mathrm{mPa}$ s), even small fall heights are sufficient for kinetic masking. More viscous media require larger falls. For example, a $0.35 \%$ alginate solution (sonicated $15 \mathrm{~min}$ ) has a viscosity of $7 \mathrm{mPa} \mathrm{s}$ in case of $L$. trabeculata and a viscosity of $6 \mathrm{mPa}$ in case of L. nigrescens. These can pass the mask after a fall of $110 \mathrm{~cm}$.

\subsection{Masking process}

Figure 2a shows a drop split by a metal mask (round holes of 1,000 $\mu \mathrm{m}$ diameter) in lateral view. The instant when the drop first hits the mask is taken as time zero $\left(t_{0}=0 \mathrm{~ms}\right)$. The drop passes through the grid with rather little broadening and separates into several liquid tubes (see Fig. 2a3). 
Fig. 2 a Characteristics of a drop division by a metal round hole mask (see a1) recorded by a high-speed video camera at 4,000 frames per second in lateral view (grid size $800 \mu \mathrm{m}$, d1 $20 \mathrm{~cm}$, cannula inner diameter $1.2 \mathrm{~mm}$ ). b Plot of the position of the leading edge of a drop $(3.1 \mathrm{~mm}$ diameter, $20 \mathrm{~cm}$ fall) before impact and the leading edge of the central tube afterwards. c Plot of the radial flow of a masked drop
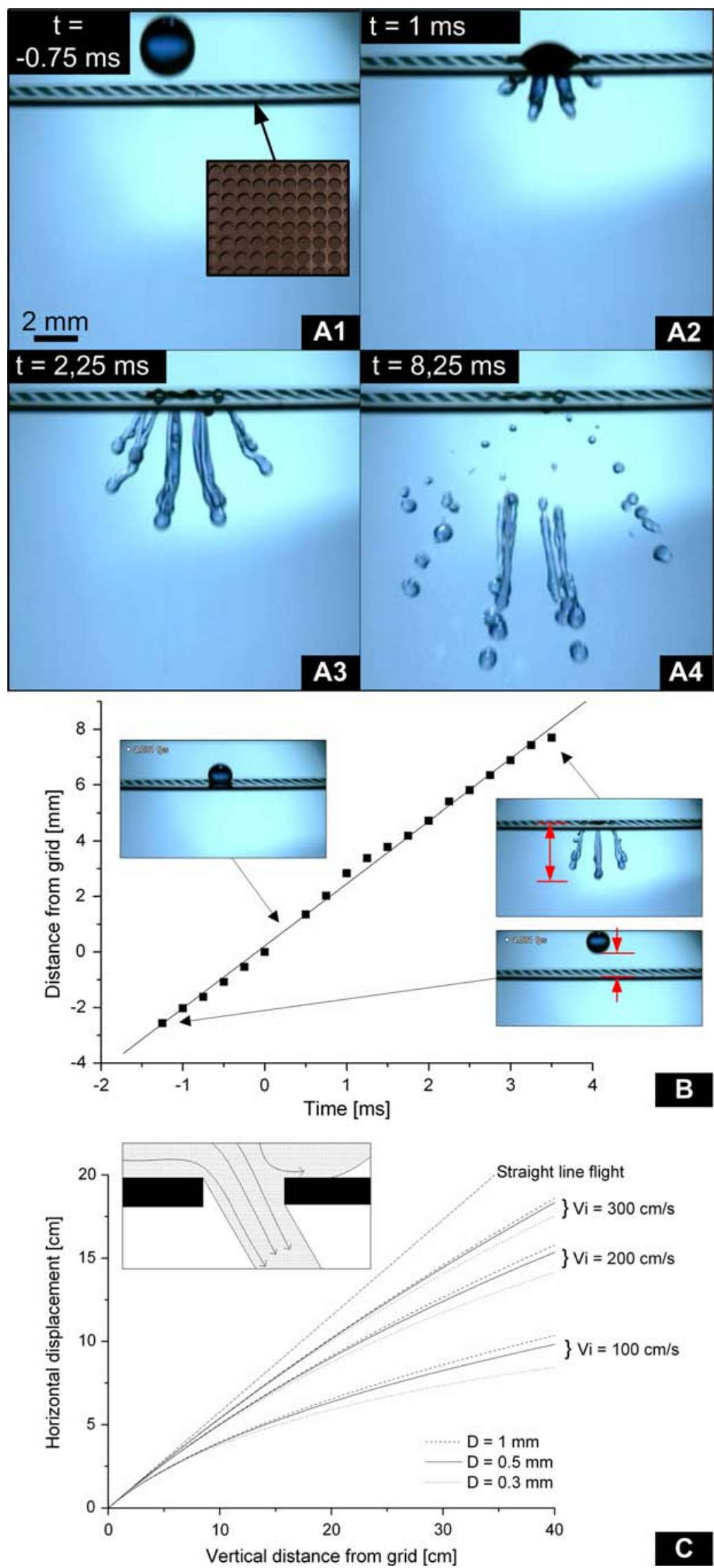
The number of tubes is the number of holes through which the drop actually passed and depends on drop diameter and grid pitch. The tubes curve outward and spread away from the centre of impact, with the outer ones deflected more than the inner.

The passage of a $1.8 \mathrm{~mm}$ diameter water drop from $d_{1}=20 \mathrm{~cm}$ through a metal mask (round hole, see Fig. 1c3) gives a tube angle $\alpha$ of $91^{\circ}$ at time $t=6.25 \mathrm{~ms}$. Increasing the height to $40 \mathrm{~cm}$ gives an angle $\alpha$ of $77^{\circ}$ at $t=2.5 \mathrm{~ms}$. The angles were measured at the times when the tips of the liquid tubes reached the same z-position. With a HDPE grid (size $800 \mu \mathrm{m}$ ), $1.8 \mathrm{~mm}$ drop and $\mathrm{d}_{1}=40 \mathrm{~cm}$, the angle was $85^{\circ}$.

Figures $2 \mathrm{a}, 3$ and 4 show the tubes breaking into microdroplets (with volumes ranging from picolitres up to about $0.3 \mu \mathrm{l}$ ), with the leading ones tending to be larger than the trailing ones. Droplet formation usually starts at about $3.5 \mathrm{~ms}$ and the tubes also appear to straighten at about this time. Before attaining their final spherical shape, many droplets can be seen to be rotating and/or oscillating.

Experiments with plasma-treated surfaces (oxygen) showed reduced contact angles of water on the masks (reduction from $87.73^{\circ}$ to $3.49^{\circ}$ ), but revealed no obvious changes in the masking process.

\subsection{Reproducibility of the separating process}

Figure 3 illustrates the high reproducibility of tube formation and Fig. 4 that of the final droplet pattern. For a reproducible pattern, the impact point must be controlled (compare Fig. 2a, where the impact point seems to be on the land of a grid, with Fig. 3a, where the impact is over a hole). But, as long as the impact point remains constant (as in Fig. 3), tube formation and thus the final droplet pattern on the substrate are identical.

The number of tubes (e.g. Fig. 3) equals the number of grid holes penetrated by the drop and the number of major spots in the resulting droplet pattern (see Fig. 4). The liquid tubes break into a multitude of droplets, but the flight vector of droplets from the same liquid tube is nearly identical, so that they finally merge again on the substrate.

The resulting droplet's volume and size mainly depend on the sample itself and on the metrics of the mask.

\subsection{Reproducibility and scaling of the resulting pattern}

Figure 4a1-c1 shows photographs of droplet patterns. The spot patterns of different runs using the same parameter set can be overlaid. Examples for the three different parameter
Fig. 3 a, b Frames of three different runs with equal parameters at the same time $t$, each of a kinetically masked ultra-pure water drop. The drops were generated by cannulas with an inner diameter of 0.4 and $1.2 \mathrm{~mm}$, respectively and separated through a metal round hole grid in (a) and a metal square grid in (b). The exposures illustrate the high reproducibility of the process

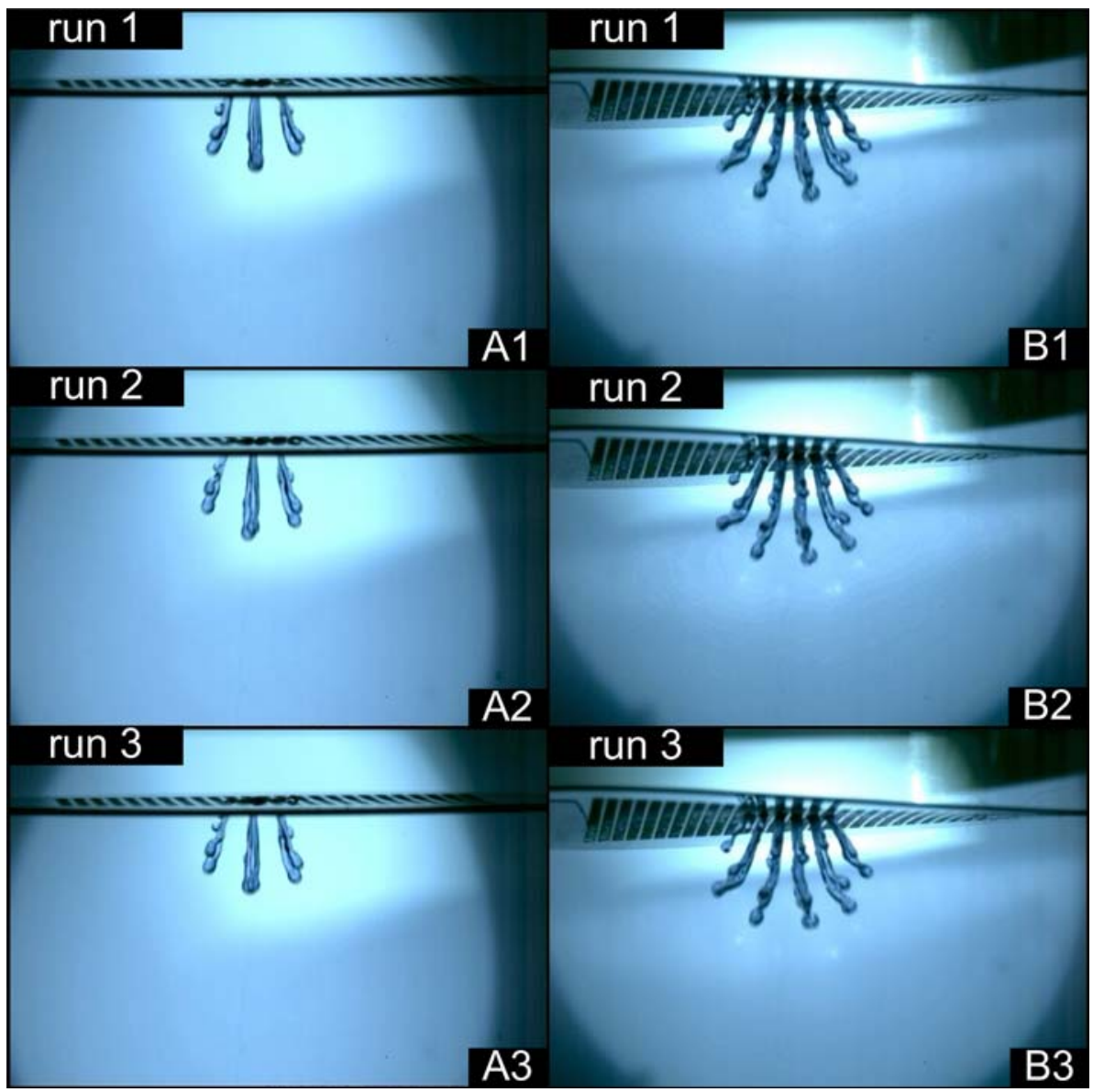


sets are shown in Fig. 4 and the reproducibility is remarkable.

The grids used for Fig. 4 were regular and the main droplets in each pattern are equidistant. The parameters of Fig. $4 \mathrm{a}, \mathrm{b}$ differ only in $d_{2}$, the distance between masking unit and target, which allows the whole pattern to be scaled up and down. However, the relationship between spot separation and $d_{2}$ is not linear.

Also, the initial drop size affects the resulting pattern. Figure $4 \mathrm{a}$, c has the same $d_{2}$, but Fig. $4 \mathrm{a}$ used a $1.8 \mathrm{~mm}$ drop and gave a spot-spot distance of about $0.75 \mathrm{~cm}$, whereas Fig. 4c used a $2.8 \mathrm{~mm}$ drop and the separation was about $0.55 \mathrm{~mm}$.

The outer spots, particularly, are elongated along the radius from the centre of the pattern. This is also the projection of the flight vector of the liquid tube.

To yield reproducible droplet patterns, it is crucial to ensure a clean drop breakaway and a central impact on the grid. If these conditions are not met, the resulting pattern can have outlying minor spots (e.g. black in Fig. 4c2). The minor spots also tend to lie along the radii.

\subsection{Applications}

\subsubsection{Masking of cell suspensions}

Dispensing cell suspensions with the kinetic mask yields excellent cell vitality, even at large fall distance $\left(d_{1}=110 \mathrm{~cm}\right)$ and small mask holes (HDPE mask with $200 \mu \mathrm{m}$ holes). With all combinations of height and mask, cell viability exceeded $99 \%$ when droplets were gathered from the target by pipette and a vitality test performed. Cells could also be cultivated in the droplet pattern. However, not all cells pass through the mask; some remain in the liquid, clinging to the mask.

\subsubsection{Encapsulation}

3D encapsulation of cell-alginate mix in general yields cell vitalities of at least $95 \%$. This excellent viability depends to some extent on the fact that the cell suspension is mixed in a 1:1 ratio with the alginate solution. Centrifugation and resuspension of cells in pure alginate reduce vitality to $85 \%$. As shown in Fig. 5a, the capsules resulting from crosslinking cell-containing alginate with a viscosity of $7 \mathrm{mPa} s$ vary in size from 300 to $2,000 \mu \mathrm{m}$ (maximum diameter) and have somewhat irregular shapes. The inset in Fig. 5a shows a fluorescently stained capsule. The cells appear to be distributed randomly with no preferred regions.

Figure 5b shows a 2D encapsulation of cells in ultrasonically treated $0.7 \%$ L. trabeculata alginate solution (treatment duration: $6 \mathrm{~min}$, viscosity: $32 \mathrm{mPa} \mathrm{s}$ ). The pattern was produced by a metal grid (hole size $800 \mu \mathrm{m}$ ) and intercepted on a poly-L-lysine treated Petri dish; the initial drop gained its kinetic energy from a $60 \mathrm{~cm}$ fall and the resulting main spots are about $2,000 \mu \mathrm{m}$ (maximum diameter). Comparison experiments were performed by spotting cells with a NanoPlotter on a similar dish. The patterns were cross-linked in $\mathrm{BaCl}_{2}$ solution. The inset of Fig. $5 \mathrm{~b} 1$ shows that the viability of encapsulated cells after kinetic masking is very high. It varies from 85 to $98 \%$ in the single droplets. In contrast, cells spotted with the plotter were not vital (not shown). The inset in Fig. 5b is a live-dead stain of a single droplet produced by kinetic masking (2D encapsulation). A single droplet produced by the Nanoplotter shows only red cells, and the vitality is always $0 \%$.

\subsubsection{Cryopreservation}

Figure 5c displays the setup for cryopreservation experiments using kinetic masking. Instead of the computerised target mount, a Petri dish was located centrally underneath the mask floating on liquid nitrogen in a dewar. High-speed video shows that the droplets reach the target dish at slightly different times (data not shown). Freezing rate can be estimated by examining the frames. Large droplets are entirely frozen in about $60 \mathrm{~ms}$, which corresponds to a rate between about 800 and $1,200^{\circ} \mathrm{C} / \mathrm{min}$. Smaller droplets freeze faster than larger ones and the tiny droplets produced by the $200 \mu \mathrm{m}$ HDPE grid freeze even before they reach the surface. The shoulder in the vertical temperature profile (Fig. 5d) corresponds to the top of the dewar, and the grid at $10 \mathrm{~cm}$ above the nitrogen surface is only about $1.6^{\circ} \mathrm{C}$ below ambient air temperature. Work is in progress to develop suitable cryo-protocols for cells.

\subsubsection{Mixing of micro-volumes}

Figure 5e1, e2 displays the effect of a wet grid on the masking process of an ultra-pure water drop, dyed with phenol red, and how this can be useful in biotechnology. A dry mask divides the drop normally (Fig. 5e1). A mask wetted with ultra-pure water intensely dyed with trypan blue seems to mix the solutions and have a drastic effect on the masking process.

\section{Discussion}

\subsection{The mask process}

The masking process is clearly complex and the possibility of an analytical or, probably, even a numeric solution, is being revised. This discussion is, therefore, limited to an estimation of the magnitude of some of the quantities involved. 
Fig. 4 Photograph of one resulting droplet pattern (1) and corresponding overlays (coloured red, blue and black) of three different runs (2) of kinetic masking with identical parameters. In each case, the sample was ultra-pure water dyed with trypan blue, and the masking process was performed with a square metal grid (grid size $800 \mu \mathrm{m})$. a Cannula with $0.4 \mathrm{~mm}$ inner diameter,

$d_{2}=15 \mathrm{~mm}$. b Cannula with

$0.4 \mathrm{~mm}$ inner diameter,

$d_{2}=25 \mathrm{~mm}$. c cannula with

$2.7 \mathrm{~mm}$ inner diameter,

$d_{2}=15 \mathrm{~mm}$

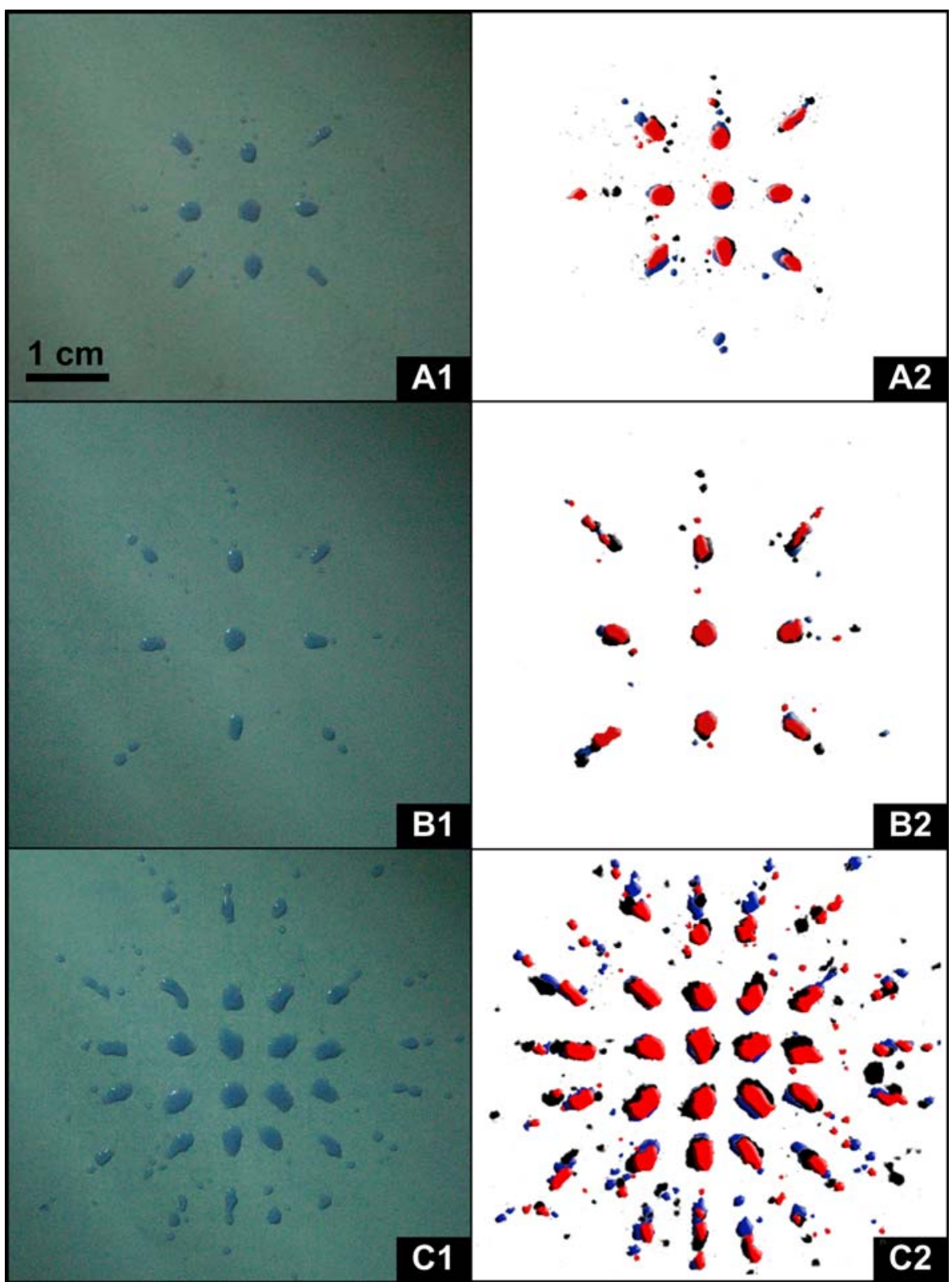

The sizes of the initial drops produced by different cannulas follow closely the equation based on the work of Heertjes et al. (1971). The velocities, measured just before impact, are as expected from the height of fall. Table 1 shows the dependence of terminal fall velocity on drop size. Even at the greatest height of fall, the impact velocity $(\sim 4.6 \mathrm{~m} / \mathrm{s})$ is only a small fraction of the terminal velocity of the smallest $(1.8 \mathrm{~mm})$ drops. A $1.8 \mathrm{~mm}$ drop, after a short $(100 \mathrm{~mm})$ fall, has kinetic energy of $\sim 30 \times 10^{-7} \mathrm{~J}$ and, if pure water, a surface energy of $\sim 12 \times 10^{-7} \mathrm{~J}$. However, for most combinations of drop size and fall height reported here, the surface energy is a smaller fraction of the total initial energy.
The video frames presented here generally cover a period of $\sim 10 \mathrm{~ms}$. In this time, the force of gravity will cause a droplet to move by only $\sim 0.5 \mathrm{~mm}$, and air resistance at sub-terminal velocities will have an even smaller effect. The motion during the impact itself is, therefore, primarily governed by inertia, viscosity and interfacial tension.

Figure $2 b$ plots the position of the leading edge of a drop (3.1 $\mathrm{mm}$ diameter, $20 \mathrm{~cm}$ fall) before impact and the leading edge of the central tube afterwards. The velocity of the leading edge is very nearly constant. However, the trailing edge of the tube moves much more slowly. The initial drop traverses its own diameter in $\sim 1.5 \mathrm{~ms}$, but the tail of the tube may take $\sim 8 \mathrm{~ms}$ to emerge from the mask. 
Fig. 5 a Photograph of a Petri dish containing microcapsules produced by the process of kinetic masking. Inset shows a typical single capsule with fluorescence-stained cells. b 2D encapsulation: Petri dish containing alginate-cell droplet pattern produced by kinetic masking with a metal grid (size $800 \mu \mathrm{m})$. Inset shows a typical single capsule with

fluorescence-stained cells. c Photograph of the kinetic masking setup used for the cryopreservation experiments. d Measured temperature gradient above the liquid nitrogen surface (setup see c). e High-speed video exposures of a masking process with a dry (e1) and a wetted (e2) metal grid to the same time point. The masking process of the wet grid is retarded and of very different characteristics

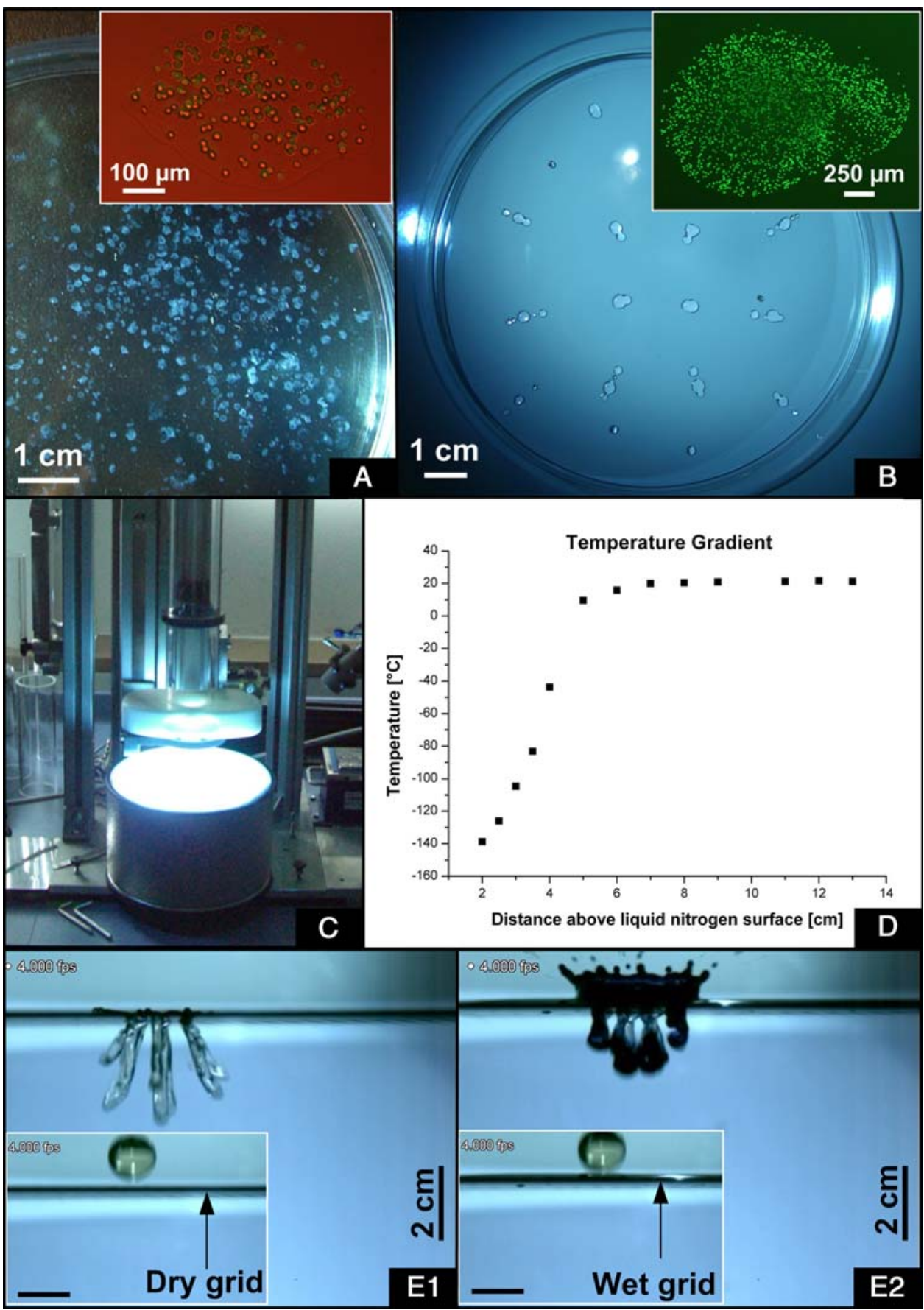

drop to travel its own diameter, which may explain the curvature of the tubes during this period, as flows are hardly likely to be constant. At this time, the residual hemispheric form of the drop disappears and vertical and horizontal velocities on the upper surface of the mask fall to low values. However, the liquid is still visible above the mask for a much longer time. It may be that after the initial impact, liquid is pulled through the holes by the momentum of the leading part of the tube. Certainly, the emerging tubes are much straighter during this subsequent period. Figure $5 \mathrm{e} 2$ shows a drop impacting on a wet grid. It displays 
Table 1 The surface energy is area $\times[\sigma-T \times(\mathrm{d} \sigma / \mathrm{d} T)]$ where $\sigma$ is the surface tension and $T$ the absolute temperature

\begin{tabular}{lccccc}
\hline $\begin{array}{l}\text { Diameter } \\
(\mathrm{mm})\end{array}$ & $\begin{array}{l}\text { Volume } \\
(\mu \mathrm{l})\end{array}$ & $\begin{array}{l}\text { Area } \\
\left(\mathrm{mm}^{2}\right)\end{array}$ & $\begin{array}{l}\text { Surface } \\
\text { energy } \\
\left(\mathrm{J} \times 10^{7}\right)\end{array}$ & $\begin{array}{l}\text { Terminal } \\
\text { velocity } \\
(\mathrm{m} / \mathrm{s})\end{array}$ & $k\left(\mathrm{~s}^{-1}\right)$ \\
\hline 3 & 14.1 & 28.3 & 33.4 & 268 & 0.0366 \\
1 & 0.524 & 3.14 & 3.70 & 29.8 & 0.329 \\
0.3 & 0.0141 & 0.283 & 0.334 & 2.68 & 3.66 \\
0.1 & 0.000524 & 0.0314 & 0.037 & 0.298 & 32.9 \\
\hline
\end{tabular}

$k=6 \pi \eta r / m=g / v_{\mathrm{t}}$ is the reciprocal of the stopping time of the particle. Where $\eta$ is the viscosity of air, $r$ the drop radius, $m$ the drop mass, $g$ the acceleration due to gravity and $v_{\mathrm{t}}$ the terminal velocity. The values have been taken from standard tables

a typical splash crown (absent in dry grids) and the emerging tubes are nearly vertical. Clearly, the spreading processes are quite different in the wet and dry cases.

The initial drop has energy $\sim 370 \times 10^{-7} \mathrm{~J}$, of which about $90 \%$ is kinetic. It is possible to make rough estimates of the total tube kinetic energy $\left(60-100 \times 10^{-7} \mathrm{~J}\right)$, tube surface energy $\left(90-140 \times 10^{-7} \mathrm{~J}\right)$ and surface energy of the water-mask interface $\left(<20 \times 10^{-7} \mathrm{~J}\right)$. The watermask interface is, therefore, a minor term in the overall energy balance, and it is not surprising that modifying the mask surface had no apparent effect on the process.

The straightening of the leading curved part of the tube cannot be attributed purely to surface tension. The surface area of a toroidal segment is identically equal to that of a straight cylinder of the same (tube) radius and volume. So, surface tension will drive the segment towards a spherical form, without a tendency to pass through an intermediate straight form. The straightening is likely to arise from a combination of surface tension and the velocity gradient that exists along the tube.

The fragmentation of a jet (with length $>$ perimeter) into droplets is a well-known phenomenon and experience suggests that high viscosity will tend to stabilise the jet.

Whilst gravity and air resistance are of little consequence during the mask impact, they are important during the subsequent flight of the droplets. Fig. 5c illustrates the likely range of trajectories for droplets of different sizes and initial velocities, initially moving at $30^{\circ}$ to the vertical. The curvature and spread of the lines is almost certainly the cause of the non-linearity of pattern scaling and the elongation of final spots, which become marked when masktarget distance exceeds about $2 \mathrm{~cm}$.

\subsection{Experimental setup}

The major factor affecting reproducibility seems to be the impact point of the drop on the grid. The anti-static precautions seem to be adequate, but the addition of heat filters for the lighting should reduce convection currents in the downpipe and help to control impact point. Also, protection of the target area from draughts should improve reproducibility.

The metallic grids seem to induce less curvature of the liquid jets than do the HDPE ones. However, this might be a consequence of aspect ratio, rather than material. Further experiments with various materials and geometries are needed.

Embedding cells in a matrix (similar to 19) might protect them, although shear forces increase with the viscosity of the medium and conditions would have to be carefully controlled. The influence on masking processes with liquids of a viscosity far above that of water has not been examined yet.

Kinetic masking is the first method that is capable of direct defined and parallel dispensing of cell suspensions, and can be used to generate arrays of micro-cell reactors. The process of masking has no major effect on the vitality of L929 cells, although there is some apparent loss of cells. The degree of loss varies with the medium, but never exceeds half. Further experiments will clarify at which point of the system this loss occurs.

Kinetic masking is limited by the viscosity of the samples and the homogeneity of the resulting droplets. Nevertheless, especially for biologically relevant solutions, this method has clear advantages. The high reproducibility and defined droplet patterns are improvements over spraying techniques. In summary, we introduced an efficient and low-cost dispenser for biologically relevant fluids, especially cell suspension, with promising applications in biotechnology, biology and medicine.

Acknowledgments This work was supported by a grant from Bundesministerium für Bildung und Forschung (BMBF) given to H. Zimmermann (grant no. 16SV2275 and 0315029A).

Open Access This article is distributed under the terms of the Creative Commons Attribution Noncommercial License which permits any noncommercial use, distribution, and reproduction in any medium, provided the original author(s) and source are credited.

\section{References}

Allain LR, Askari M, Stokes DL, Vo-Dinh T (2001) Microarray sampling-platform fabrication using bubble-jet technology for a biochip system. Fresenius J Anal Chem 371:146-150. doi: 10.1007/s002160100962

Allain LR, Stratis-Cullum DN, Vo-Dinh T (2004) Investigation of microfabrication of biological sample arrays using piezoelectric and bubble-jet printing technologies. Anal Chim Acta 518:7785. doi:10.1016/j.aca.2004.04.065

Chin CD, Linder V, Sia SK (2007) Lab-on-a-chip devices for global health: past studies and future opportunities. Lab Chip 7:41-57. doi:10.1039/b611455e 
Cooley P, Wallace D, Antohe B (2002) Applicatons of ink-jet printing technology to BioMEMS and microfluidic systems. JALA 7:3339. doi:10.1016/S1535-5535(04)00214-X

de Heij B, Steinert C, Sandmaier H, Zengerle R (2003) A tuneable and highly parallel picolitre-dispenser based on direct liquid displacement. Sens Actuators A Phys 103:88-92. doi:10.1016/ S0924-4247(02)00321-7

Ehrhart F, Gepp MM, Howitz S, Zimmermann H (2007) Selective 2D alginate encapsulation of adherent cells for regenerative medicine with a novel nanoplotter. 12. Leipziger workshop cytomics and translational medicine 2007 : incorporating: 5th International workshop. Slide Based Cytometry 71:528-529

Grohn P, Klock G, Zimmermann U (1997) Collagen-coated Ba(2+)alginate microcarriers for the culture of anchorage-dependent mammalian cells. Biotechniques 22:970-975

Gustavsson P, Johansson F, Kanje M, Wallman L, Linsmeier CE (2007) Neurite guidance on protein micropatterns generated by a piezoelectric microdispenser. Biomaterials 28:1141-1151. doi: 10.1016/j.biomaterials.2006.10.028

Gutmann O, Niekrawietz R, Kuehlwein R, Steinert CP, de Heij B, Zengerle R, Daub M (2004) Impact of medium properties on droplet release in a highly parallel nanoliter dispenser. Sens Actuators A Phys 116:187-194. doi:10.1016/j.sna.2004.04.021

Heertjes PM, de Nie LH, de Vries HJ (1971) Drop formation in liquid-liquid systems-II testing of the considerations given in Part I, for drop volumes below the jetting velocity: a criterion for the jetting velocity. Chem Eng Sci 26:451-459. doi:10.1016/ 0009-2509(71)83018-X

Hillgärtner $M$, Zimmermann $H$, Mimietz S, Jork A, Thürmer F, Schneider H, Nöth U, Hasse C, Haase A, Fuhr G, Rothmund M, Zimmermann U (1999) Immunoisolation of transplants by entrapment in 19F-labelled alginate gels: production, biocompatibility, stability, and long-term monitoring of functional integrity. Materialwiss Werkstofftech 30:783-792. doi:10.1002/ (SICI)1521-4052(199912)30:12<783::AID-MAWE783>3.0.CO; 2-\#

Koltay P, Steger R, Bohl B, Zengerle R (2004) The dispensing well plate: a novel nanodispenser for the multiparallel delivery of liquids (DWP Part I). Sens Actuators A Phys 116:483-491. doi: 10.1016/j.sna.2004.05.038

Laurell T, Wallman L, Nilsson J (1999) Design and development of a silicon microfabricated flow-through dispenser for on-line picolitre sample handling. J Micromech Microeng 9:369-376. doi: 10.1088/0960-1317/9/4/314

Lin SC, Tseng FG, Huang HM, Huang CY, Chieng CC (2001) Microsized 2D protein arrays immobilized by micro-stamps and micro-wells for disease diagnosis and drug screening. Fresenius $\mathbf{J}$ Anal Chem 371:202-208. doi:10.1007/s002160100991

Okamoto T, Suzuki T, Yamamoto N (2000) Microarray fabrication with covalent attachment of DNA using bubble jet technology. Nat Biotechnol 18:438-441. doi:10.1038/74507

Roth EA, Xu T, Das M, Gregory C, Hickman JJ, Boland T (2004) Inkjet printing for high-throughput cell patterning. Biomaterials 25:3707-3715. doi:10.1016/j.biomaterials.2003.10.052

Thielecke H, Impidjati, Zimmermann H, Fuhr GR (2005) Gentle cell handling with an ultra-slow instrument: creep-manipulation of cells. Microsyst Technol 11:1230-1241. doi:10.1007/s00542005-0584-7

Zimmermann H, Hagedorn R, Fuhr G (2002) Method and device for dosing fluid media, WO/2002/102515, Germany

Zimmermann H, Wahlisch F, Baier C, Westhoff M, Reuss R, Zimmermann D, Behringer M, Ehrhart F, Katsen-Globa A, Giese C, Marx U, Sukhorukov VL, Vasquez JA, Jakob P, Shirley SG, Zimmermann U (2007) Physical and biological properties of barium cross-linked alginate membranes. Biomaterials 28:13271345. doi:10.1016/j.biomaterials.2006.11.032 